\title{
Estimation Accuracy of Ejection Fraction in Gated Cardiac SPECT/CT Imaging using Iterative Reconstruction with 3D Resolution Recovery in Rapid Acquisition Protocols
}

\author{
Johannes Zeintl*, Student Member, IEEE, Xinhong Ding, A. Hans Vija*, Eric G. Hawman, Joachim Hornegger, and \\ Torsten Kuwert, Members, IEEE
}

\begin{abstract}
Iterative reconstruction methods with 3D resolution recovery, and attenuation and scatter compensations are now common in clinical practice. Still, the reconstruction of ECG gated cardiac SPECT data is often done with filtered backprojection (FBP) to save reconstruction time. The reconstruction times of the 2007 release of Flash3D (Siemens' OSEM reconstruction with 3D distance dependent resolution recovery and optional scatter and attenuation corrections) have been significantly improved and it is now possible to process an entire clinical gated cardiac data set in clinically acceptable times. It has been shown that Flash3D enables the use of rapid acquisition protocols, yet maintains clinical diagnostic ability. In this work we evaluated the estimation accuracy of the cardiac ejection fraction of gated myocardial SPECT/CT perfusion images, acquired with currently used clinical protocols, as well as rapid acquisition protocols.

For this, we acquired ECG gated image data of a dynamic cardiac torso phantom manufactured by Data Spectrum. Images were reconstructed using FBP and the new Flash3D with and without scatter and attenuation corrections. We analyzed the reconstructed volumes of the dynamic phantom using our own quantitative analysis tool as well as 4D-MSPECT and compared the image-based estimated ejection fraction (EF) to the true ejection fraction delivered by the dynamic phantom. Results show high correlation $\left(r^{2}>0.97\right)$ between conventional clinical protocols with FBP reconstruction and half time protocols using Flash3D except for 16 gates an $128 \times 128$ matrix $\left(r^{2}=0.95\right)$. Best ejection fraction accuracy was found for 16 gates and $64 \times 64$ matrix with a mean deviation of $0.5 \pm 1.2 \%$ (full time FBP) $0.9 \pm 1.6 \%$ (half time Flash3D) from the true value.
\end{abstract}

Manuscript received November 23, 2007. Asterisk indicates corresponding author.

*Johannes Zeintl is a $\mathrm{PhD}$ student with the University of ErlangenNuremberg, Institute of Pattern Recognition, Erlangen, Germany. (e-mail: Johannes.Zeintl@nuklear.imed.uni-erlangen.de).

*Dr. A. Hans Vija (e-mail: hans.vija@siemens.com), Dr. Xinhong Ding, Dr. Eric G. Hawman are with Siemens Medical Solution USA, Inc., Molecular Imaging, Hoffman Estates, IL 60192, USA.

Prof. Dr. Joachim Hornegger is with the University of Erlangen-Nürnberg, Institute of Pattern Recognition, Erlangen, Germany.

Prof. Dr. Torsten Kuwert is with the University of Erlangen-Nürnberg, Clinic of Nuclear Medcine

\section{INTRODUCTION}

TERATIVE reconstruction methods including corrections for image degrading effects such as collimator blur and photon attenuation and scattering are now common in clinical practice, in part due to the availability of fast computers at low cost, and advances in efficient processing methods. Still, the reconstruction of ECG gated cardiac SPECT data is often done with filtered backprojection (FBP) to save reconstruction time.

The reconstruction times of the 2007 release of Flash3D (Siemens' OSEM reconstruction with 3D distance dependent resolution recovery and optional scatter and attenuation corrections) have been significantly improved. It is now possible to process an entire clinical gated cardiac data set in less than one minute. Table I shows the reconstruction times per iteration of the latest release of Flash3D for typical clinical data dimensions.

Previously, it has been shown, on the basis of static phantom data in combination with clinical studies, that Flash3D enables the use of rapid acquisition protocols, yet maintains clinical diagnostic ability providing sufficient myocardial uniformity and lesion detectability [6]. In this work we focus on the estimation accuracy of the cardiac ejection fraction of gated myocardial SPECT/CT perfusion images. Currently used clinical protocols, are compared to $50 \%$ time-optimized acquisition protocols.

Common current quantitative cardiac packages for the processing of gated SPECT data were ultimately designed for current clinical protocols [1]-[4]. In addition to using one of them for the evaluation of half time images, we also developed our own estimation tool for comparison. 
TABLE I

RECONSTRUCTION TIMES OF FLASH3D 2007 RELEASE (INTEL XEON 51502 CPU DUAL-CORE, 2.66 GHz, 3 GB RAM)

\begin{tabular}{cccc}
\hline \hline \multicolumn{2}{c}{ Typical Data Dimensions } & \multicolumn{2}{c}{ Time/Iteration } \\
\hline $\begin{array}{c}\text { Reconstructed } \\
\text { Volume }\end{array}$ & Projection Data & Uncorrected & Corrected $^{*}$ \\
\hline $64 \times 64 \times 52$ & $64 \times 64 \times 64$ & $0.099 \mathrm{~s}$ & $0.11 \mathrm{~s}$ \\
$128 \times 128 \times 104$ & $128 \times 128 \times 64$ & $0.61 \mathrm{~s}$ & $0.76 \mathrm{~s}$ \\
\hline \hline
\end{tabular}

*both scatter and attenuation correction applied within the iteration loop

\section{Materials AND MethodS}

\section{A. Data Acquisition and Processing}

To avoid reliance on Monte Carlo simulation, which only approximates a real clinical SPECT system, we use an actual system with a physical dynamic phantom. The phantom employed is the Dynamic Cardiac Phantom (both genders) manufactured by Data Spectrum, an anthropomorphic torso phantom with liver, lung, and spine inserts (Figure 1 top). The left ventricular bloodpool and myocardium are mimicked by two latex membranes connected to an electric pump which simulates the blood flow and also provides an ECG trigger signal, in order to perform gated acquisitions. The package includes a user interface to control the pump (Figure 1 bottom). End-diastolic volume (EDV), end-systolic volume (ESV), and heart beat can be specified. In addition, the phantom software allows to 'freeze' the motion at 8 discrete positions of the cardiac cycle (see Figure 1 bottom, positions F2-F9). Fig. 2 shows example CT images of the phantom in these positions. For SPECT acquisitions the phantom was loaded with ${ }^{99 \mathrm{~m}}$ Technetium adjusting the activity concentration ratio of heart:liver:background to 14:8:1. High count ECG gated data sets with dense angular and spatial sampling were acquired in a dual headed SPECT/CT system (Symbia $^{\mathbb{B}}$ T2/T6). Gated timeslots, matrix size, and ejection fraction were varied according to Table II.

From this measured data ("reference"), we created "whatif" datasets. For this we developed a DICOM compatible tool that extracts angular views from a projection data set, rebins the matrix and pixel size and reduces counts in a pixel by a selected fraction using binomial subsampling while maintaining the Poisson character of the data [5]. In this way, we create gated projection data sets with different levels of counts and angular sampling for the assessment of clinical and rapid protocols. For the purpose of this work we focused on the common clinical protocol (according to ASNC recommendation [7]) in comparison to a half time protocol generated by extraction of every other projection angle $\left(3^{\circ} \rightarrow\right.$ $6^{\circ}$ angular sampling) [6]. The myocardial count density in the projection data was approximately $4.5 \mathrm{cts} / \mathrm{cm}^{2}$. In order to increase statistics, five realizations for each data set shown in Table II for both clinical as well as half time count level were created using the binomial subsampling technique mentioned earlier (160 generated data sets total). In addition to the gated acquisitions static high count SPECT/CT acquisitions of separate gates (phantom positions F2-F9) were acquired and processed in terms of counts and spatial and angular resolution as mentioned above. The data were reconstructed using FBP and OSEM-3D (Flash3D).

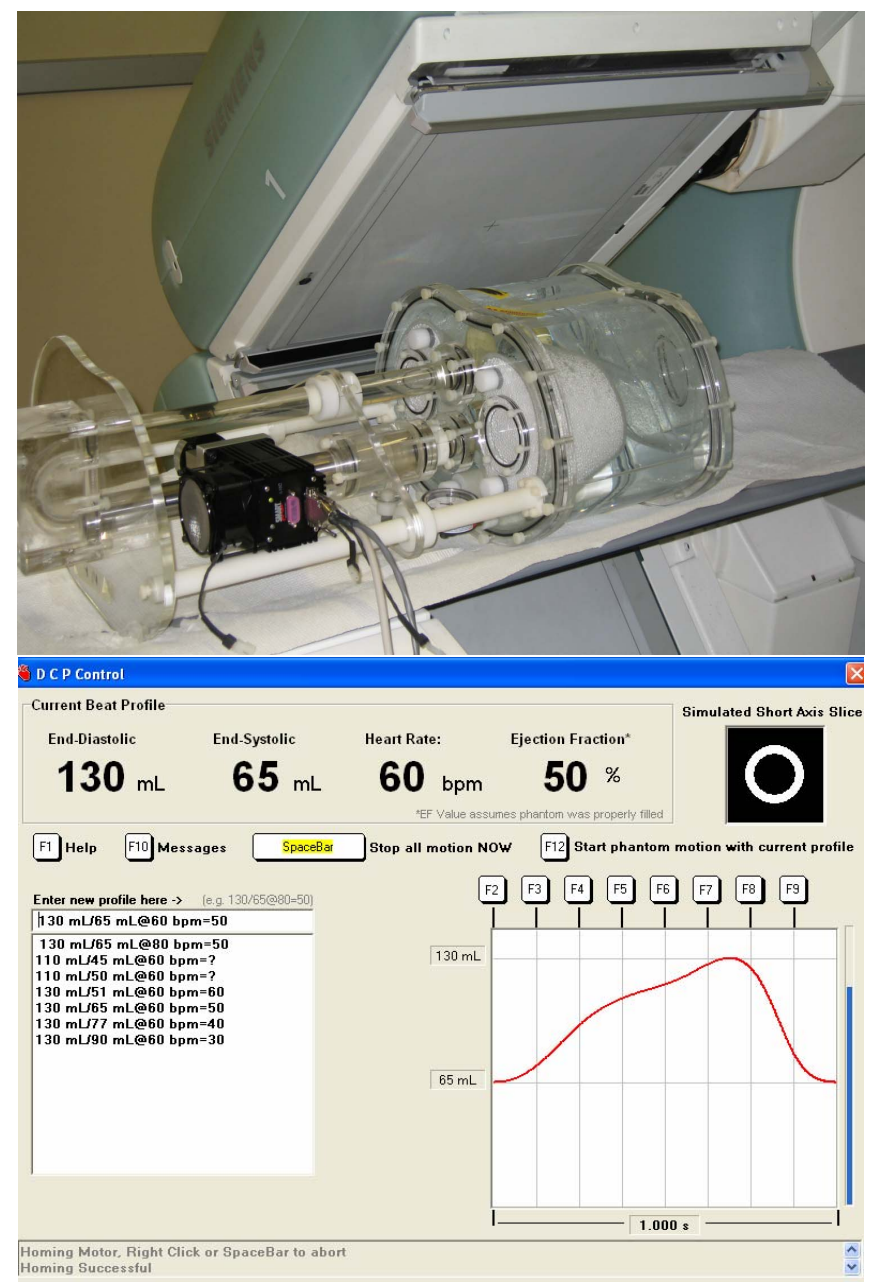

Fig. 1. Dynamic Cardiac Phantom (top) manufactured by Data Spectrum and provided software to control cardiac parameters (bottom). The software allows for adjusting ESV, EDV, EF, and heart rate. In addition the phantom can be stopped at 8 discrete positions of the cardiac cycle (F2-F9).

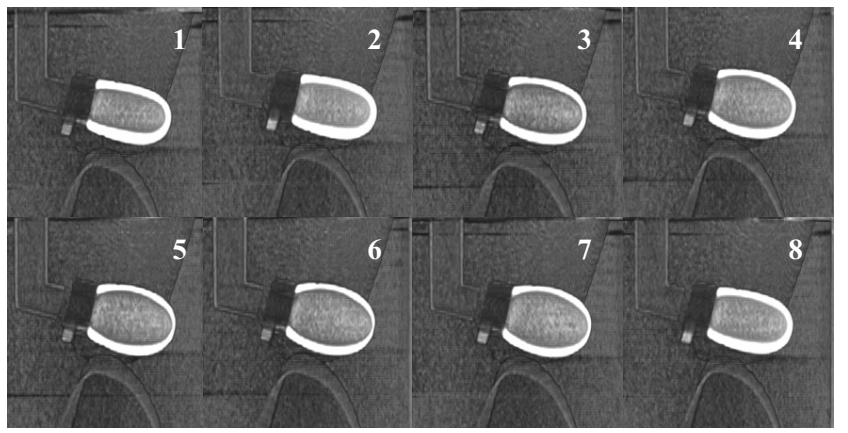

Fig. 2. CT images of the dynamic phantom stopped at positions F2-F9 (see Fig. 1 bottom). Volume parameters were set according to Fig. 1 bottom. For visualization purposes $\mathrm{CT}$ contrast agent was injected into the myocardial chamber. 
TABLE II

GATED ACQUISITION

PARAMETERS

\begin{tabular}{ccc}
\hline Matrix Size & \# of Timeslots & Ejection Fraction (\%) \\
\hline \multirow{2}{*}{128} & 8 & $30,40,50,60$ \\
& 16 & $30,40,50,60$ \\
\hline \multirow{2}{*}{64} & 8 & $30,40,50,60$ \\
& 16 & $30,40,50,60$ \\
\hline
\end{tabular}

\section{B. Image Analysis}

Reconstructed gated data was analyzed in terms of ESV, EDV, and EF using 4D-MSPECT (MedImage, Ann Arbor, Michigan), one of the major currently used quantitative cardiac analysis packages.

In addition, a new measurement tool for estimating the absolute bloodpool volumes as well as the ejection fraction was developed. This tool was specifically designed for the dynamic phantom using the known phantom geometry.

The myocardial segmentation and the bloodpool volume estimation are performed slice by slice on the short axis (SA) images of the reconstructed volumes. The main steps for the image based bloodpool volume estimation are illustrated in Figure 3.

The initial segmentation of the myocardium is done by applying a threshold of $40 \%-50 \%$ of the maximum pixel value to the image slice. After thresholding, the image may still contain unwanted regions, especially if the image is noisy (see Fig. 3). To optimize the segmentation, a region cleanup is performed by applying a combination of region labeling, using prior knowledge of the myocardial shape, and morphological operations.

In addition to the segmentation a phantom specific model is introduced to facilitate the volume estimation. Since the phantom simulates the myocardium with two cylindrical membranes, a simple circular model is fitted to the segmented myocardium in the short axis slices. For a given set of $\mathrm{n}$ points $\left(x_{i}, y_{i}\right): 1 \leq i \leq n$ representing the $2 \mathrm{D}$ positions of the segmented myocardial region in a short axis slice, the least square estimate of the radius of the best circle for this region is calculated according to the following equation:

$$
\begin{gathered}
\hat{r}_{L S}=\frac{\sum_{i}\left(x_{i}-x_{0}\right) \cos \left(\theta_{i}\right)+\sum_{i}\left(y_{i}-y_{0}\right) \sin \left(\theta_{i}\right)}{n} \\
\theta_{i}=\arctan \left(\frac{y_{i}}{x_{i}}\right)
\end{gathered}
$$

where $\left(\mathrm{x}_{0}, \mathrm{y}_{\mathrm{o}}\right)$ denotes the center coordinates of the circle.

The radius of the bloodpool used for the volume calculation is then derived by subtracting half of the known myocardial wall thickness.

Using a model based approach in addition to the thresholding shows its benefits especially in the case of high variations in pixel intensity caused e.g. by attenuation effects or 'blobby' images due to low signal to noise ratios.

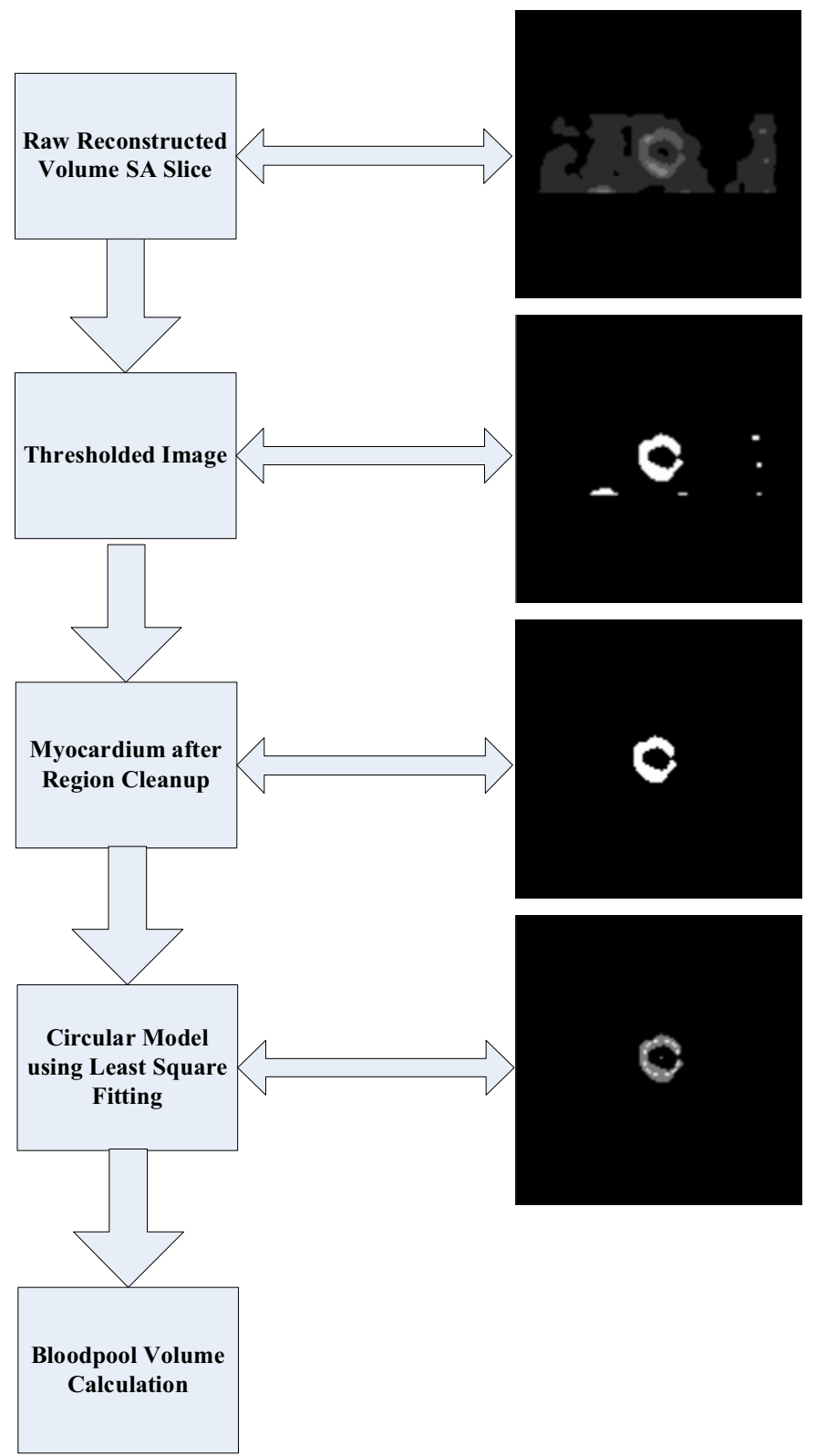

Fig. 3. Main steps for image based bloodpool volume estimation - a combination of thresholding and circular model calculation using prior knowledge of the phantom geometry.

Since bloodpool volume estimation is very sensitive towards the setting of the base slice, a base slice calibration was performed on both estimation tools. Fig. 4 gives a general overview of the procedures for tool calibration, volume estimation and verification using the phantom's gold standard. For the localization of the base slice the SPECT data was superimposed with the hardware registered CT volume provided by the hybrid scanner. The base slice was then used as a constant parameter in the estimation tools. The assumption of a constant base slice location could be justified, since there was no physical translation of the phantom's 
myocardium during the scan. The truth model of the bloodpool volumes and therewith the ejection fraction was derived by two independent measurements. Both image based volumetric analysis of cardiac bloodpool studies of the phantom were performed as well as physical measurements of the mechanical pump displacement (cp. Fig. 4).

SPECT/CT Registration Obtaining Base Slice

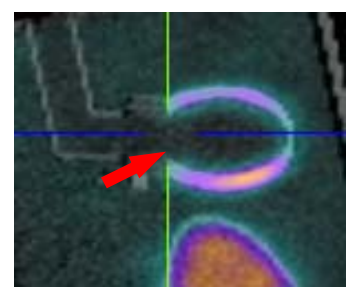

Base Slice Calibration

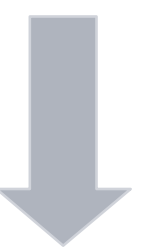

Left Ventricular Volume Estimation

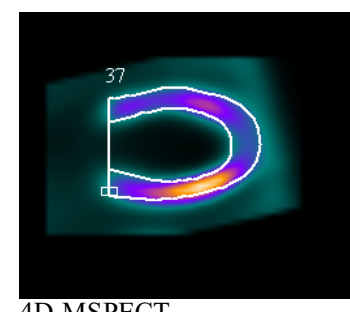

4D-MSPECT

Comparison to expected value

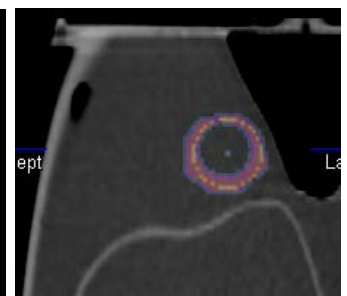

Internally developed tool

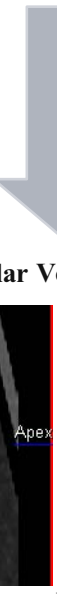

Bloodpool study image analysis

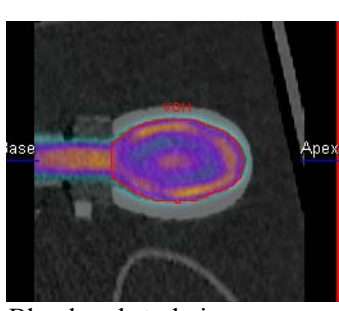

Mechanical pump displacement
Fig. 4. Overview of procedures for tool calibration, volume estimation and verification using the phantom's gold standard.

\section{RESULTS}

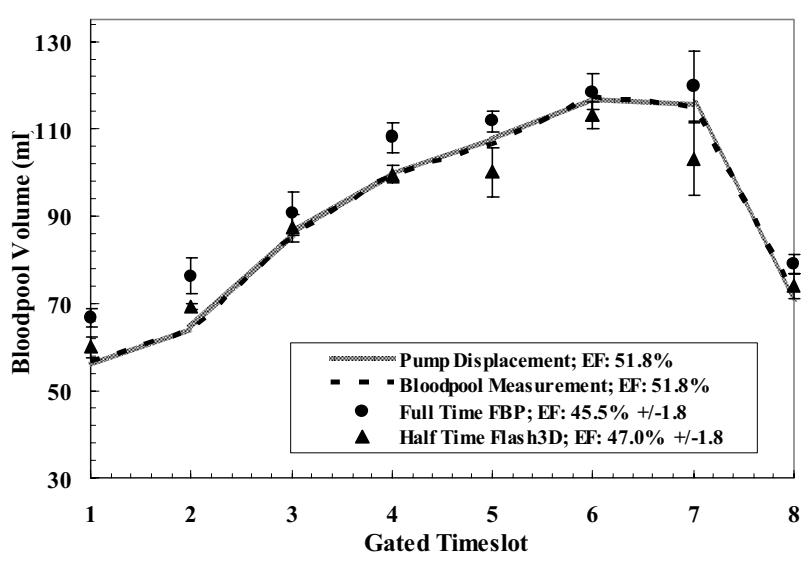

Fig. 5. Absolute volumes of separately acquired gates estimated with internally developed measurement tool - Comparison of full time FBP protocol versus half time Flash3D. Error bars shown are standard deviations.

TABLE III

MEAN EJECTION FRACTION ACCURACY AND CORRELATION (GATED ACQUISITION DATA)

\begin{tabular}{ccccc}
\hline \hline \# Time slots & Matrix size & \multicolumn{2}{c}{ Mean deviation from true value (\%) } & $\begin{array}{c}\text { Correlation Coefficient } \\
\text { (FT FBP vs. HT } \\
\text { Flash3D) }\end{array}$ \\
\hline \multirow{2}{*}{8} & FT FBP & HT Flash3D & 0.9769 \\
& 64 & $-2.6 \pm 3.0$ & $0.8 \pm 3.0$ & 0.9891 \\
\multirow{2}{*}{16} & 128 & $-4.2 \pm 1.0$ & $-4.7 \pm 0.8$ & 0.9825 \\
& 64 & $0.5 \pm 1.2$ & $0.9 \pm 1.6$ & 0.9515 \\
\hline \hline
\end{tabular}

TABLE IV

TRUE NORMALIZED DIFFERENCE FROM TRUE EJECTION FRACTION VALUE (16 TIME SLOTS 64X64)

\begin{tabular}{ccccc}
\hline \hline & \multicolumn{2}{c}{ Full Time } & \multicolumn{2}{c}{ Half Time } \\
& Flash3D & FBP & Flash3D & FBP \\
\hline$\Delta$ EF (\%) & $0.9 \pm 2.6$ & $1.6 \pm 2.7$ & $2.1 \pm 3.0$ & $3.2 \pm 2.9$ \\
$\Delta$ EDV (\%) & $-7.6 \pm 1.9$ & $-4.3 \pm 1.6$ & $-7.6 \pm 1.8$ & $-4.0 \pm 2.6$ \\
$\Delta \mathbf{~ E S V ~ ( \% ) ~}$ & $-8.3 \pm 3.8$ & $-4.9 \pm 3.8$ & $-9.1 \pm 4.2$ & $-6.1 \pm 3.4$ \\
\hline \hline
\end{tabular}

Note: Values are normalized to the true value respectively. 

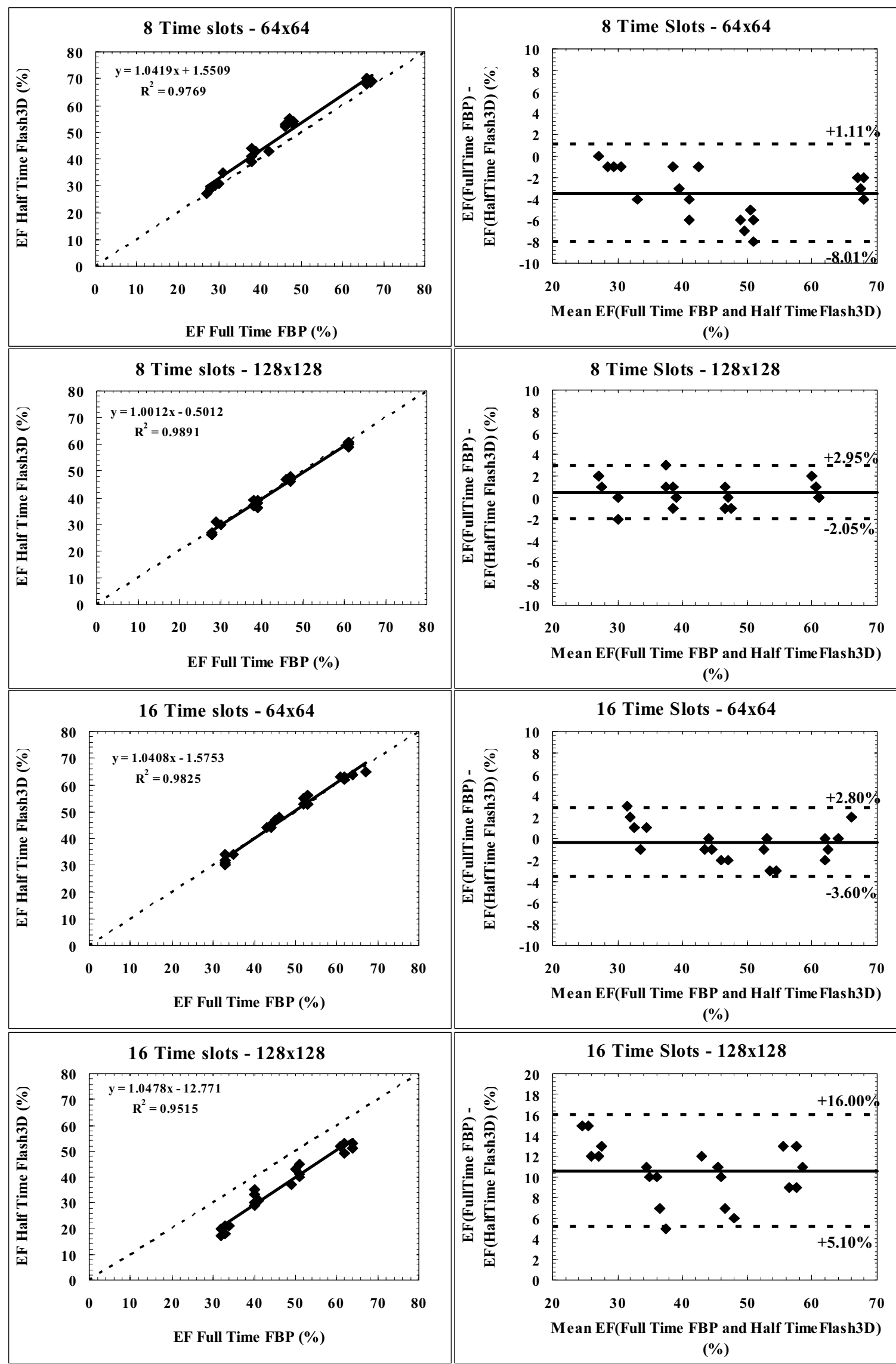

Fig. 6. Phantom data: Correlation Analysis (left column) and Band-Altmann Plots (right column) of full time FBP versus half time Flash3D gated datasets (cp. Table II). EF estimation was done with 4D-MSPECT. 


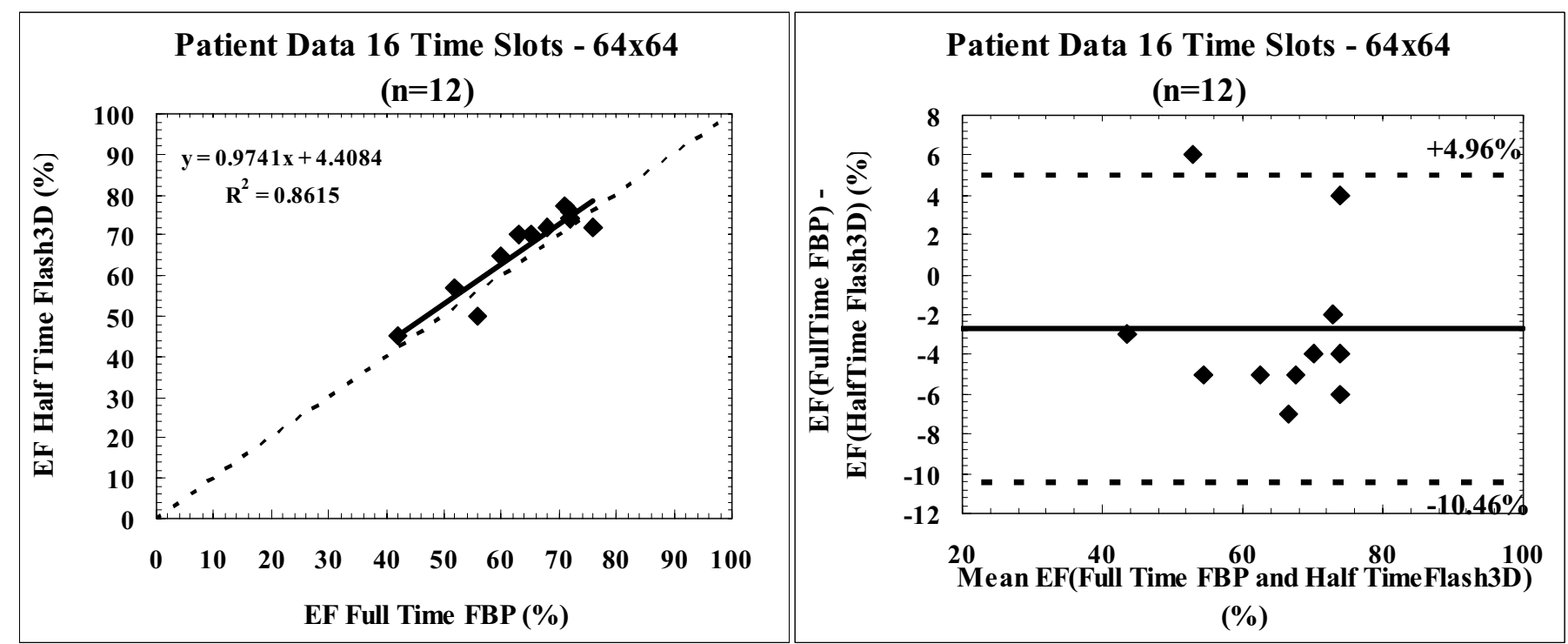

Fig. 7. Patient data verification: Correlation analysis (left) and Band-Altmann Plot of a pilot set of 12 patients (gated stress data acquired at the Iowa Heart Center, De Moines, Iowa). Half time projection data were generated retrospectively; Imaging parameters: 64/32 total views (FT/HT), approx. 4.5 cts/mm2, 16 time slots, $64 \times 64$ matrix, reconstruction parameters according to phantom study.

\section{Discussion}

4D-MSPECT is not designed for static volume estimation, nor for phantoms. Hence, we developed our own tool. The model to estimate EF may be sub-optimal for this phantom data, but the described calibration procedure yields satisfactory results. Results of volume and EF estimation depend on both reconstruction technique and estimation tool. Using 4D-MSPECT errors in volumes EDV and ESV are somewhat greater with Flash3D reconstruction than with FBP, yet errors in EF are smaller. The standard deviations for FBP and Flash $3 \mathrm{D}$ are in the same range. The volume estimation error may be due to sub-optimal 4D-MSPECT performance for this data set. The EF values of FT FBP and HT Flash3D correlate well $\left(\mathrm{r}^{2}>0.97\right)$, except for 16 gates and $128 \times 128$ matrix, which also show a $-12 \%$ bias in EF due to an underestimation of EDV by $16 \%$ and overestimation of ESV by $4 \%$. Best agreement with true EF in HT imaging results from data with 16 gates and 64x64 using Flash3D, however it is not yet statistically significant at this noise level and 5 realizations. More work is needed to decide if differences are statistically significant.

\section{CONCLUSION}

EF values are robust towards changes in count level or reconstruction technique. Using iterative reconstruction with 3D resolution recovery (Flash3D) and 4D-MSPECT the estimation accuracy of ejection fraction in gated cardiac SPECT/CT imaging in half time acquisition (HT) protocols is comparable to standard acquisition (FT) protocol using 16 gates and $64 \times 64$ matrix with $6.6 \mathrm{~mm}$ pixels. Clinical reconstruction times of Flash3D are fast and thus Flash3D can be used for the entire processing of either FT or HT studies. 